\title{
An Empirical Model of Angle-of-Arrival Variance for a Gaussian Wave Propagation through Non-Kolmogorov Turbulence
}

\author{
Chao Gao $\mathbb{D}^{1,2,3}$ Libin Jiao, ${ }^{1,2}$ and Xiaofeng $L i \mathbb{C}^{3}$ \\ ${ }^{1}$ The 54th Research Institute of China Electronics Technology Group Corporation, Shijiazhuang 050081, China \\ ${ }^{2}$ Science and Technology on Information Transmission and Dissemination in Communication Networks Laboratory, \\ Shijiazhuang 050081, China \\ ${ }^{3}$ School of Astronautics and Aeronautic, University of Electronic Science and Technology of China, Chengdu 611731, China \\ Correspondence should be addressed to Xiaofeng Li; lxf3203433@uestc.edu.cn
}

Received 4 September 2019; Accepted 25 April 2020; Published 26 May 2020

Academic Editor: Sulaiman W. Harun

Copyright (C) 2020 Chao Gao et al. This is an open access article distributed under the Creative Commons Attribution License, which permits unrestricted use, distribution, and reproduction in any medium, provided the original work is properly cited.

\begin{abstract}
This paper proposes an empirical model of the angle-of-arrival (AOA) variance for a Gaussian wave propagating through the weak non-Kolmogorov turbulence. The proposed model is approximately expressed as the linear weighted average between the AOA variances of the plane and spherical waves. The Monte Carlo method is applied to validate the proposed model. The numerical simulations indicate that, under the geometrical optics approximation, the AOA variance for a Gaussian wave is insensitive to the change of the diffraction parameter and can be closely approximated by a simple linear relationship in the refraction parameter. These two properties ensure the validity of the empirical model.
\end{abstract}

\section{Introduction}

Optical wireless communication technology has attracted widespread attention during the past few decades. It adopts unguided laser beam propagating in random media to carry the digital signal for data transmission with high data rates. The random media, however, may contain numerous invisible turbulent eddies, which fluctuate the refractive index inside the random media and distort the equiphase wavefront of the laser beam. Considering that the equiphase wavefront in the vacuum is undistorted and uniform, AOA can be defined as the angle between the normal vectors of the distorted and undistorted equiphase wavefront. It is generally accepted that AOA could hardly degenerate the quality of the imaging spot, but will lead the focus position to deviate from the optimal point. This phenomenon is equivalent to the geometric misalignment between the receiving and transmitting antennas [1-3].

AOA stochastically fluctuates with the changes in both temporal and spatial dimensions, and the degree of the AOA fluctuation is conventionally described by its variance. In recent years, theoretical models of the AOA variances for plane and spherical waves have been fully analyzed based on different turbulent power spectrums [4-15]. However, the research on the AOA variance for a Gaussian wave still needs further discussion. Gao et al. [16] proposed a heuristic model of the AOA variance for a Gaussian wave propagation through non-Kolmogorov turbulence, but lacked adequate evidence to ensure the validity of the expressions. Regarded as an increment to the previous literature studies, this paper not only investigates the similar scenario but also conducts simulations for experimental verification. The rest of the paper is organized as follows. Section 2 presents the theoretical models of the AOA variances for different types of laser beams. In Section 3, numerical simulations are performed, followed by conclusions in Section 4 .

\section{Theoretical Models}

2.1. AOA Variances for Plane and Spherical Waves. The AOA variance for either plane or spherical wave can be calculated by

$$
\sigma_{\mathrm{AOA}}^{2}=\frac{D_{S}(d)}{(k d)^{2}}
$$


where $d$ is the aperture diameter of the collecting lens, $k=$ $2 \pi / \lambda$ is the angular wavenumber with the wavelength $\lambda$, and

$$
D_{S}(\rho)=4 \pi^{2} k^{2} L \int_{0}^{1} \mathrm{~d} \xi \int_{0}^{+\infty} d \kappa \times \kappa \Phi_{n}(\kappa) f(\kappa, \xi),
$$

is the phase structure function [1]. In equation (2), $\rho$ is the scalar separation between two observation points, $L$ is the propagation optical path length, $\xi$ is the normalized path coordinate, and $\kappa$ is the scalar spatial wavenumber which regularized the scale of the turbulence eddy. $\Phi_{n}(\kappa)$ and $f(\kappa, \xi)$ in equation (2) represent the turbulent power spectrum and the weight function, respectively. This paper applies the benchmark non-Kolmogorov model:

$$
\Phi_{n}(\kappa)=A(\alpha) \hat{C}_{n}^{2} \kappa^{-\alpha},
$$

as the turbulent power spectrum, where $\alpha \in(3,4)$ is the general spectral power law value, $A(\alpha)=\Gamma(\alpha-1) / 4 \pi^{2} \cos \alpha \pi / 2$ is a function related to $\alpha$, $\Gamma(z)=\int_{0}^{+\infty} x^{z-1} e^{-x} \mathrm{dx}$ is the gamma function, and $\widehat{C}_{n}^{2}$ is the scalar generalized atmospheric structure parameter [14]. The weight function for the plane wave $f_{p}(\kappa, \xi)$ and for the spherical wave $f_{s}(\kappa, \xi)$ are defined as

$$
\left\{\begin{array}{l}
f_{p}(\kappa, \xi)=\left(1-J_{0}(\kappa \rho)\right) \times\left(1+\cos \left(\frac{L \kappa^{2}}{k} \xi\right)\right), \\
f_{s}(\kappa, \xi)=\left(1-J_{0}(\kappa \xi \rho)\right) \times\left(1+\cos \left(\frac{L \kappa^{2}}{k} \xi(1-\xi)\right)\right),
\end{array}\right.
$$

where $J_{0}(x)=\sum_{n=0}^{+\infty}(-1)^{n} /(n !)^{2}(x / 2)^{2 n}$ is the Bessel function of the first kind with zero order.

To achieve the closed-form expression of equation (1), this paper invokes the geometrical optics approximation (GOA) to reduce equation (2). For terrestrial horizontal wireless optical communication link, the Fresnel length $l_{F}=$ $\sqrt{\lambda L}$ is usually in the order of magnitude of centimeter, whereas $d$ is designed in the order of magnitude of decimeter, i.e., $d>l_{F}$ is almost satisfied. For $\rho>l_{F}$, the lens effect of the turbulent eddy can be dealt with GOA, which yields $L \kappa^{2} / k \ll 1$. Consequently, equation (4) leads to

$$
\left\{\begin{array}{l}
f_{p}(\kappa, \xi) \approx 2\left(1-J_{0}(\kappa \rho)\right) \\
f_{s}(\kappa, \xi) \approx 2\left(1-J_{0}(\kappa \xi \rho)\right)
\end{array}\right.
$$

Based on the equation for $-3<p<-1$ and $a>0$ [17]:

$$
\int_{0}^{+\infty} x^{p}\left(1-J_{0}(a x)\right) d x=-a^{-p-1} 2^{p} \Gamma\left(\frac{1+p}{2}\right) \Gamma\left(\frac{1-p}{2}\right)^{-1},
$$

the double integrals in equation (2) can be reduced to

$$
\left\{\begin{array}{l}
\int_{0}^{1} d \xi \int_{0}^{+\infty} d \kappa \times \kappa \Phi_{n}(\kappa) f_{p}(\kappa, \xi) \approx-A(\alpha) \widehat{C}_{n}^{2} \rho^{\alpha-2} 2^{2-\alpha} \times \Gamma\left(\frac{2-\alpha}{2}\right) \Gamma\left(\frac{\alpha}{2}\right)^{-1} \\
\int_{0}^{1} d \xi \int_{0}^{+\infty} d \kappa \times \kappa \Phi_{n}(\kappa) f_{s}(\kappa, \xi) \approx-\frac{1}{\alpha-1} A(\alpha) \widehat{C}_{n}^{2} \rho^{\alpha-2} 2^{2-\alpha} \times \Gamma\left(\frac{2-\alpha}{2}\right) \Gamma\left(\frac{\alpha}{2}\right)^{-1} .
\end{array}\right.
$$

Thus, based on GOA, the AOA variances for plane and spherical wave can be rewritten as

$$
\left\{\begin{array}{l}
\sigma_{\mathrm{AOA}, p}^{2} \approx-\pi^{2} \mathrm{LA}(\alpha) \hat{C}_{n}^{2} d^{\alpha-4} 2^{4-\alpha} \times \Gamma\left(\frac{2-\alpha}{2}\right) \Gamma\left(\frac{\alpha}{2}\right)^{-1}, \\
\sigma_{\mathrm{AOA}, s}^{2} \approx \frac{\sigma_{\mathrm{AOA}, p}^{2}}{\alpha-1} .
\end{array}\right.
$$

It is found that under GOA, the AOA variances are independent to $\lambda$. This property has been confirmed under various situations $[15,16,18]$.

2.2. AOA Variances for a Gaussian Wave. The Gaussian beam is a representative type of the electromagnetic wave, with normally distributed transverse electric field and intensity [19]. The mathematical description of a Gaussian wave usually depends on the wavelength $\lambda$ and the waist radius $w_{0}$. For the optical wireless communication, more parameters are necessary to determine the location of both the transmitter and the receiver. The curvature parameter $\Theta_{0}=1-L / R_{0}$ and the Fresnel ratio $\Lambda_{0}=2 L / k W_{0}^{2}$, two nondimensional scalars, are related to the transmitter, where $R_{0}$ is the radius of curvature of the phase wavefront at the transmitter and $W_{0}$ is the radius where the intensity reduces to $1 / e^{2}$ of the axial values at the transmitter. Similarly, there are also three nondimensional scalars, the refraction parameter $\Theta=\Theta_{0} / \Theta_{0}^{2}+\Lambda_{0}^{2}$, the complementary parameter $\bar{\Theta}=1-\Theta$, and the diffraction parameter $\Lambda=\lambda L / \pi W^{2}$, related to the receiver, where $W=W_{0} \sqrt{\Theta_{0}^{2}+\Lambda_{0}^{2}}$ is the beam radius in a vacuum at the receiver.

It is known that both plane and spherical waves are particular types of the Gaussian waves; therefore, their AOA variances should take the form as follows: 


$$
\left\{\begin{array}{l}
\sigma_{\mathrm{AOA}, p}^{2}=\sigma_{\mathrm{AOA}, G}^{2}(\Lambda=0, \Theta=1), \\
\sigma_{\mathrm{AOA}, s}^{2}=\sigma_{\mathrm{AOA}, G}^{2}(\Lambda=0, \Theta=0) .
\end{array}\right.
$$

For the given Gaussian wave with arbitrary values of $\Lambda$ and $\Theta$, it is difficult to achieve the analytic formulae of $\sigma_{\mathrm{AOA}, G}^{2}$. As an alternative, $\sigma_{\mathrm{AOA}, \mathrm{G}}^{2}$ may be approximatively expressed by $\sigma_{\mathrm{AOA}, p}^{2}$ and $\sigma_{\mathrm{AOA}, s}^{2}$. On the one hand, according to the definition of $\Lambda$, it follows that

$$
\lim _{\lambda \longrightarrow 0} \Lambda=0 .
$$

On another hand, equation (8) implies that, under GOA, both $\sigma_{\mathrm{AOA}, p}^{2}$ and $\sigma_{\mathrm{AOA}, s}^{2}$ are independent to $\lambda$. Thus, a heuristic inference can be made that $\sigma_{\mathrm{AOA}, G}^{2}$ may be insensitive to the change of $\Lambda$ when $d>l_{F}$, i.e.,

$$
\sigma_{\mathrm{AOA}, \mathrm{G}}^{2} \approx \sigma_{\mathrm{AOA}, \mathrm{G}}^{2}(\Lambda=0) .
$$

Furthermore, $\sigma_{\mathrm{AOA}, G}^{2}(\Lambda=0)$ may be closely approximated by a simple linear relationship in $\Theta$, i.e.,

$$
\sigma_{\mathrm{AOA}, G}^{2}(\Lambda=0) \approx \Theta \sigma_{\mathrm{AOA}, p}^{2}+(1-\Theta) \sigma_{\mathrm{AOA}, s}^{2} .
$$

Equation (12) is inspired by [20], which investigated the on-axis scintillation index for a Gaussian wave in the saturation regime.

\section{Numerical Simulations}

This section adopts the Monte Carlo method to validate equations (11) and (12), respectively. In our simulations, the distorted equiphase wavefront is generated by the stochastic phase screen with the first 496 terms of the Zernike polynomials, whose theoretical formulae are presented in Section 4.1. Afterwards, over 50 points are stochastically sampled on the surface of the distorted equiphase wavefront, and the corresponding AOA can be computed by their normal vectors. For each combination of optical parameters, the simulations are repeated 100 times, and the AOA variance can be statistically estimated by the empirical data. Other default settings are listed as follows: $\alpha \in\{3.2,3.5,3.8\}$, $\widehat{C}_{n}^{2}=1.0 \times 10^{-15} \mathrm{~m}^{3-\alpha}, \quad \lambda=1.55 \times 10^{-6} \mathrm{~m}, \quad k \approx 4.05 \times 10^{6}$ $\mathrm{rad} / \mathrm{m}, L=4000 \mathrm{~m}$, and $d=0.1 \mathrm{~m}>l_{F}=0.078 \mathrm{~m}$.

To validate equation (12), $\Lambda$ is fixed at 0 , whereas $\Theta$ is assigned to 11 points linearly spaced between and including 0 and 1. Particularly, $\Theta=1$ refers to the plane wave, and $\Theta=0$ refers to the spherical wave. The simulation results are depicted in Figure 1, where the dots stand for the experimental values, and the lines stand for the theoretical values. It can be identified that there is linear relationship between $\sigma_{\mathrm{AOA}, G}^{2}(\Lambda=0)$ and $\Theta$. Thus, equation (12) is valid within the range of the allowable error.

To validate equation (11), $\Theta$ is fixed at 0.5 . Considering that the optical wireless communication systems usually adopt the collimators to parallel the outgoing beam at the transmitting antenna, $\Lambda$ is assigned to 11 points linearly spaced between and including 0 and 0.5 . Figure 2 illustrates the simulation results. It is evident that due to the observational errors, the experimental values of $\sigma_{\mathrm{AOA}, G}^{2}$ fluctuate around the theoretical values of $\sigma_{\mathrm{AOA}, G}^{2}(\Lambda=0)$, and the

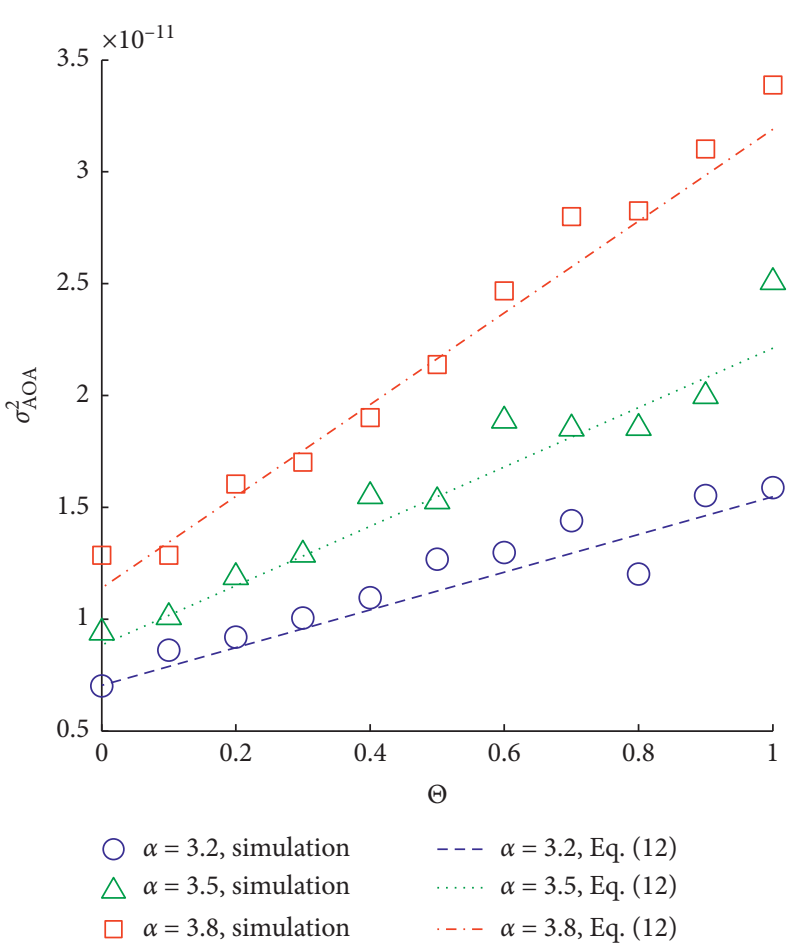

FIGURE 1: Validation of equation (12).

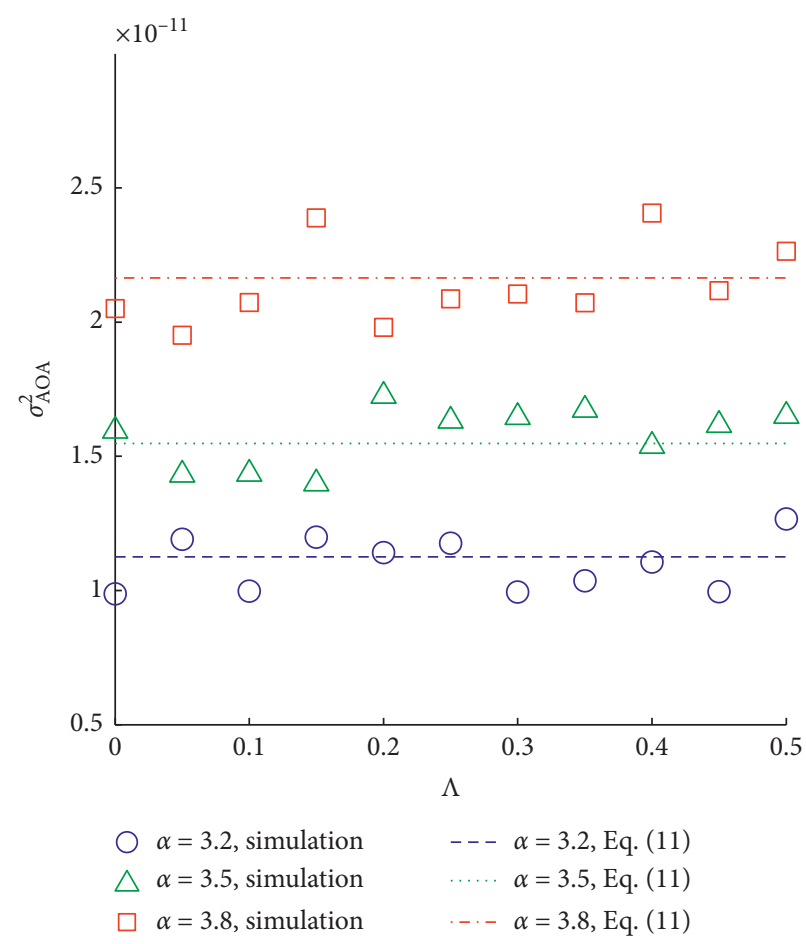

FIgURE 2: Validation of equation (11).

margin of the relative error is less than $15 \%$. Thus, equation (11) is valid for $d>l_{F}$. This phenomenon can be explained physically. The validity of equation (11) is associated with the validity of equation (10). The condition " $d>l_{F}$ " ensures the validity of GOA, which implies that diffraction effects are 
negligible and can be generally characterized by the limiting case with $\lambda \longrightarrow 0$. Hence, equation (10) is valid under GOA.

\section{Conclusions}

This paper investigates the AOA variance for a Gaussian wave propagating through the weak non-Kolmogorov turbulence along a horizontal link. An empirical model is deduced from the results of plane and spherical waves under the GOA. The stochastic phase screens based on the Zernike terms are generated to validate the proposed model. The numerical simulations indicate that, under the GOA:

(1) The AOA variance for a Gaussian wave is insensitive to the change of the diffraction parameter

(2) The AOA variance for a Gaussian wave can be closely approximated by a simple linear relationship in the refraction parameter

These two points ensure the validity of our proposed model.

It should be pointed out that the phase screen based on the Zernike terms is capable of characterizing the low-frequency component, but is insufficient to describe the highfrequency component. However, the high-frequency component, associated with the small-scale turbulent eddies, makes little contribution to the phase fluctuation of laser beam. Considering that AOA is directly connected with the phase fluctuation, the forementioned weakness could hardly invalidate the conclusions.

4.1. Stochastic Phase Screen with Zernike Polynomials. The Zernike polynomials are a set of binary functions orthogonal on the unit disk, whose expressions in the polar coordinate system take the form [21]:

$$
\begin{aligned}
Z_{j}(\rho, \theta) & =Z_{n, m}(\rho, \theta) \\
& =\sqrt{n+1} R_{n, m}(\rho) A_{m}(\theta),
\end{aligned}
$$

where $j=(n, m)$ is the index ordered by Noll's sequence, $\rho$ is the radius, and $\theta$ is the azimuth. $R_{n, m}(\rho)$ is the radial component:

$$
\begin{aligned}
& R_{n, m}(\rho) \\
& =\sum_{k=0}^{n-m / 2} \frac{(-1)^{k}(n-k) !}{k !(n+m / 2-k) !(n-m / 2-k) !} \rho^{n-2 k},
\end{aligned}
$$

and $A_{m}(\theta)$ is the angular component:

$$
A_{m}(\theta)= \begin{cases}\sqrt{2} \cos m \theta, & m>0 \\ \sqrt{2} \sin m \theta, & m<0 \\ 1, & m=0\end{cases}
$$

Let $\Psi$ be the distorted equiphase wavefront. It can be decomposed as $[22,23]$

$$
\Psi=\sum_{j=1}^{+\infty} c_{j} Z_{j},
$$

where $c_{j}$ are undetermined coefficients. To generate the stochastic phase screen, the covariance between $c_{j 1}$ and $c_{j 2}$ should be

$$
\operatorname{cov}\left(c_{j 1}, c_{j 2}\right)=B(\alpha)\left(\frac{d}{r_{0}}\right)^{\alpha-2} N_{n 1, n 2} M_{m 1, m 2} \times(-1)^{\frac{n 1+n 2-m 1-m 2}{2}},
$$

where $j 1=(n 1, m 1), j 2=(n 2, m 2), r_{0}$ is the Fried coherent length, and

$$
B(\alpha)=\frac{2^{3-\alpha}}{\alpha}\left(\frac{8}{\alpha-2} \Gamma\left(\frac{2}{\alpha-2}\right)\right)^{\alpha-2 / 2} \times \Gamma\left(\frac{\alpha}{2}\right) \Gamma^{-1}\left(-\frac{\alpha}{2}\right) \Gamma(\alpha+1) .
$$

$N_{n 1, n 2}$ is the factor related to the radial degrees:

$$
\begin{aligned}
N_{n 1, n 2}= & \Gamma\left(\frac{n 1+n 2-\alpha+2}{2}\right) \\
& \times \Gamma^{-1}\left(\frac{n 1+n 2+\alpha+4}{2}\right) \\
& \times \Gamma^{-1}\left(\frac{n 1-n 2+\alpha+2}{2}\right) \\
& \times \Gamma^{-1}\left(\frac{n 2-n 1+\alpha+2}{2}\right) \\
& \times \sqrt{(n 1+1)(n 2+1)},
\end{aligned}
$$

and $M_{m 1, m 2}$ is the logical Kronecker symbol related to the azimuthal frequencies:

$$
M_{m 1, m 2}=((\bmod (j 1-j 2,2)=0) \vee(m 1 m 2=0)) \wedge(m 1=m 2) \text {. }
$$

The covariance matrix $C$ about $\left\{c_{j}\right\}$ is real and symmetrical, so there must be a unitary matrix $U$ such that $S=\mathrm{UCU}^{T}$ is diagonal. $U$ can be obtained by the singular value decomposition. $R$ is a Gaussian random variable, with zero mean and variance given by $S$. The components of the vector $U^{T} R$ are the desired Zernike coefficients $c_{j}$.

The influences of $\Theta$ and $\Lambda$ on the stochastic phase screen can be delivered by $r_{0}$. In the weak fluctuation region [24],

$$
r_{0}=2.1\left(-\frac{4(\alpha-1) \Gamma(\alpha / 2)}{\pi^{2} A(\alpha) \widehat{C}_{n}^{2} k^{2} L \Gamma(1-\alpha / 2)}\right)^{1 / \alpha-2} \times\left(2^{5-\alpha} b+(\alpha-2) \Gamma\left(\frac{\alpha}{2}\right) \Lambda^{\frac{\alpha}{2}}\right)^{1 / 2-\alpha}
$$


where

$$
b= \begin{cases}\frac{1-\Theta^{\alpha-1}}{1-\Theta}, & \Theta \geq 0, \\ \frac{1+|\Theta|^{\alpha-1}}{1-\Theta}, & \Theta<0 .\end{cases}
$$

4.2. Tangent Plane and Normal Vector. The equiphase wavefront generated by the stochastic phase screen can be regarded as a surface $S$ in the three-dimensional space. Mathematically, $S$ can be described by the implicit function

$$
F(x, y, z)=0
$$

If these partial derivatives $\partial F / \partial x, \partial F / \partial y$ and $\partial F / \partial z$ are continuous over $S$, and

$$
\left(\frac{\partial F}{\partial x}\right)^{2}+\left(\frac{\partial F}{\partial y}\right)^{2}+\left(\frac{\partial F}{\partial z}\right)^{2} \neq 1
$$

$S$ is called smooth.

For arbitrary point $P_{0}=\left(x_{0}, y_{0}, z_{0}\right) \in S$, its tangent plane is

$$
\frac{\partial F}{\partial x}\left(P_{0}\right) \times\left(x-x_{0}\right)+\frac{\partial F}{\partial y}\left(P_{0}\right) \times\left(y-y_{0}\right)+\frac{\partial F}{\partial z}\left(P_{0}\right) \times\left(z-z_{0}\right)=0,
$$

and the corresponding normal line takes the form as follows:

$$
\frac{\left(x-x_{0}\right)}{\partial F / \partial x\left(P_{0}\right)}=\frac{\left(y-y_{0}\right)}{\partial F / \partial y\left(P_{0}\right)}=\frac{\left(z-z_{0}\right)}{\partial F / \partial z\left(P_{0}\right)} .
$$

The normal vector $\vec{n}=\left(\partial F / \partial x\left(P_{0}\right), \partial F / \partial y\left(P_{0}\right), \partial F / \partial z\left(P_{0}\right)\right)$ is orthogonal to the tangent plane.

For the stochastic phase screen with the Zernike polynomials, the equiphase wavefront takes the form as

$$
\Psi(x, y)-z=0
$$

with the normal vector $\vec{n}=(\partial \Psi / \partial x, \partial \Psi / \partial y,-1)$. Particularly, $\overrightarrow{n_{0}}=(0,0,-1)$ is the normal vector of the undistorted equiphase wavefront.

Since AOA can be defined as the angle between the normal vector of the distorted and undistorted equiphase wavefront, it follows that

$$
\begin{aligned}
\angle A O A & =\arccos \frac{\vec{n} \cdot \overrightarrow{n_{0}}}{|\vec{n}| \cdot\left|\overrightarrow{n_{0}}\right|} \\
& =\arccos \frac{1}{\sqrt{(\partial \Psi / \partial x)^{2}+(\partial \Psi / \partial y)^{2}+1}} .
\end{aligned}
$$

\section{Data Availability}

The data used to support the findings of this study are available within the article.

\section{Conflicts of Interest}

The authors declare that there are no conflicts of interest regarding the publication of this paper.

\section{Acknowledgments}

The authors want to thank Lingling SU from Chengdu CORPRO Technology Co., Ltd., Lingjun LI from Chengdu Technology University, and Yueting ZHOU from Patent Examination Cooperation Jiangsu Center of the Patent Office, CNIPA.

\section{References}

[1] L. C. Andrews and R. L. Phillips, Laser Beam Propagation through Random Media, SPIE Press, Bellingham, WA, USA, 2nd edition, 2005.

[2] A. Mansour, R. Mesleh, and M. Abaza, "New challenges in wireless and free space optical communications," Optics and Lasers in Engineering, vol. 89, no. 1, pp. 95-108, 2017.

[3] H. Kaushal and G. Kaddoum, "Optical communication in space: challenges and mitigation techniques," IEEE Communications Surveys \& Tutorials, vol. 19, no. 1, pp. 57-96, 2017.

[4] L. Cui and L. Cao, "Angle of arrival fluctuations of optical waves considering finite turbulence outer scale under anisotropic turbulence," Optik, vol. 127, no. 8, pp. 3820-3827, 2016.

[5] W. Du, J. Yang, Q. Gong et al., "Angle of arrival fluctuations for optical waves propagating through joint moderate to strong atmospheric turbulence," Journal of Russian Laser Research, vol. 37, no. 1, pp. 82-90, 2016.

[6] L. Cui, B. Xue, and F. Zhou, "Generalized anisotropic turbulence spectra and applications in the optical waves' propagation through anisotropic turbulence," Optics Express, vol. 23, no. 23, pp. 30088-30103, 2015.

[7] L. Cui and B. Xue, "Influence of asymmetry turbulence cells on the angle of arrival fluctuations of optical waves in anisotropic non-Kolmogorov turbulence," Journal of the Optical Society of America A, vol. 32, no. 9, pp. 1691-1699, 2015.

[8] L. Cui, B. Xue, X. Cao, and F. Zhou, "Angle of arrival fluctuations considering turbulence outer scale for optical waves' propagation through moderate-to-strong non-Kolmogorov turbulence," Journal of the Optical Society of America A, vol. 31, no. 4, pp. 829-835, 2014.

[9] L. Cui, X. Cao, B. Xue, and L. Cao, "Analysis of angle-ofarrival fluctuations for optical waves propagating through weak non-Kolmogorov turbulence," Optics Communications, vol. 305, no. 5, pp. 36-41, 2013.

[10] B. Cao, L. Cui, W. Xue, X. Bai, and F. Zhou, "Theoretical expressions of the angle-of-arrival variance for optical waves propagating through non-Kolmogorov turbulence," Optics Express, vol. 19, no. 9, pp. 8433-8443, 2011.

[11] I. Toselli, B. Agrawal, and S. Restaino, "Light propagation through anisotropic turbulence," Journal of the Optical Society of America A, vol. 28, no. 3, pp. 483-488, 2011.

[12] L. Tan, W. Du, and J. Ma, "Effect of the outer scale on the angle of arrival variance for free-space-laser beam corrugated by non-Kolmogorov turbulence," Journal of Russian Laser Research, vol. 30, no. 6, pp. 552-559, 2009.

[13] W. Du, S. Yu, L. Tan, J. Ma, Y. Jiang, and W. Xie, “Angle-ofarrival fluctuations for wave propagation through non- 
Kolmogorov turbulence," Optics Communications, vol. 282, no. 5, pp. 705-708, 2009.

[14] I. Xie, L. C. Andrews, R. L. Phillips, and V. Ferrero, "Free space optical system performance for a Gaussian beam propagating through non-Kolmogorov weak turbulence," IEEE Transactions on Antennas and Propagation, vol. 57, no. 6, pp. 1783-1788, 2009.

[15] I. Toselli, L. C. Andrews, and R. L. Phillips, "Angle of arrival fluctuations for free space laser beam propagation through non-Kolmogorov turbulence," in Proceedings of SPIE-The International Society for Optical Engineering, Orlando, FL, USA, May 2007.

[16] C. Gao, Y. Li, M. Miao, H. Liang, and X. Li, "A heuristic model of aperture-averaged angle-of-arrival variance for a Gaussian wave through anisotropic non-Kolmogorov turbulence," in Proceedings of SPIE Fourth International Conference on Optical and Photonics Engineering, Belgium, China, April 2016.

[17] I. S. Gradshteyn and I. M. Ryzhik, Table of Integrals, Series, and Products, Academic Press, Waltham, MA, USA, 8th edition, 2014.

[18] Y. Cheon and A. Muschinski, "Closed-form approximations for the angle-of-arrival variance of plane and spherical waves propagating through homogeneous and isotropic turbulence," Journal of the Optical Society of America A, vol. 24, no. 2, pp. 415-422, 2007.

[19] C. Gao and X. Li, "Effects of inner and outer scale on beam spreading for a Gaussian wave propagating through anisotropic non-Kolmogorov turbulence," Optica Applicata, vol. 47, no. 1, pp. 63-74, 2017.

[20] L. C. Andrews, M. A. Al-Habash, C. Y. Hopen, and R. L. Phillips, "Theory of optical scintillation: Gaussian beam wave model," Waves in Random Media, vol. 11, no. 3, pp. 271-291, 2001.

[21] R. J. Noll, "Zernike polynomials and atmospheric turbulence*," Journal of the Optical Society of America, vol. 66, no. 3, pp. 207-211, 1976.

[22] Y. Li, W. Zhu, and R. Rao, "Simulation of random phase screen of non-Kolmogorov atmospheric turbulence," Infrared and Laser Engineering, vol. 45, no. 12, pp. 162-169, 2016.

[23] N. A. Roddier, "Atmospheric wavefront simulation using Zernike polynomials," Optical Engineering, vol. 29, no. 10, pp. 1174-1180, 1990.

[24] S. Kotiang and J. Choi, "Wave structure function and longexposure MTF for laser beam propagation through nonKolmogorov turbulence," Optics \& Laser Technology, vol. 74, no. 1, pp. 87-92, 2015. 